\title{
Depression in Chinese Adolescents from the Perspective of Cultural Differences: A Current Review
}

\author{
Yuling $\mathrm{He}^{1, \mathrm{a},{ }^{*}, \dagger}$, Kangyi Peng ${ }^{2, \mathrm{~b},{ }^{*}, \dagger}$, Jingyi Tian ${ }^{3, \mathrm{c}, ~},+\dagger$ \\ ${ }^{1}$ Delia School of Canada, Hong Kong, China \\ ${ }^{2}$ Department of Psychology, University of Washington, Seattle, Washington, United States \\ ${ }^{3}$ University of Vermont, Burlington, Vermont, United States \\ *Corresponding author.email: ${ }^{*}$ g1014984923@gmail.com, ${ }^{b}$ pengkangyi98@gmail.com,cjtian1@uvm.edu \\ These authors contributed equally.
}

\begin{abstract}
With the increasing rate of having depression at a young age, the World Health Organization has called on worldwide concern for teenage mental health. Especially in nations with limited access to psychotherapy, experiencing mental disorders during adolescence exerts a more adverse influence on development. This paper focuses on depression in Chinese adolescents from the angle of cultural differences, including national policies. It explores the impact of Chinese culture on the formation of specialties of depression in Chinese adolescents. The paper finds that cultural differences have contributed to distinct etiology, manifestation, gender difference, and treatments of depression through a culturally accepted mechanism that remains unknown through the literature review. Compared to depression in the West, biological factors, romantic relationships, and internet addiction lead to a higher prevalence of depression in Chinese teenage girls than boys. Filial piety and the only child policy also affect Chinese teenagers exclusively. Nuances originate from culture and cannot be ignored for the development of depression. Future research should figure out the exact mechanism which outputs the Chinese adolescents' depression. This review appeals to public knowledge and psychological insight of mental health in school, family, and national education.
\end{abstract}

Keywords: Depression, Chinese Adolescents, Cultural Differences.

\section{INTRODUCTION}

Now depression has become a very common disease. About one in ten people suffer from depression. Depression affects your feeling, thinking, and acting, which causes really hard mental illness. Moreover, those who suffer from major depression may even be threatened with life due to their annoyance. Especially in the teenage years, they are vulnerable to the influence of external pressure, unable to properly regulate the psychological. Hence, they are more likely to suffer from depression and have a higher risk [1]. If not found in time and professional treatment, it will affect their mental health and even threaten their life safety. There may be more and more abundant research on depression in the West. However, there are still many contradictions and differences between the East and the West, specifically reflected in the limits of psychotherapy, cultural difference, gender discrimination, technology, etc. These contradictions and their causes will be explained in detail in this article.
Psychologists have been probing the impact of culture on mental states for a long time. The word "multiculturalism" existing in psychopathology signifies that psychologists have recognized that culture influences the etiology and manifestations of mental disorders. Although many branches of psychology, like cultural psychology and cross-cultural psychology, have focused on Eastern dependent value and Western independent value, Cohen (2009) claims that more than one form of culture should be considered while studying the cultural influence [2]. For example, the existing concept of culture is significantly related to religion, socioeconomic status, and region of the country. Cohen (2009) also proposes that culture also affects moral judgement, relationality, and so on [2]. In Gada's study (1982), sub-culture also matters as scientists found that even within India, Eastern patients with depression have different symptomatology from Western patients with depression [3]. Gada (1982) expresses that psychiatrists could detect the determinants of certain mental disorders that belong to specific groups with higher efficiency and 
accuracy [3]. The practical role of studying culture is to make education, organization, and health systems more humanized [2]. Given the above through figuring out how the Chinese culture affects depression, scientists could improve the prevention and treatments of the disorder and suggest beneficial policies which are more suitable for Chinese adolescents.

In this report, we would explore depression among Chinese teenagers and cultural differences. This report uses the literature review method to reference different articles to explain this issue. First of all, the report would talk about the fundamental thing about teenagers' depression and depression rate. And the purpose why we should study it. Secondly, the report talks about other factors that influence depression, such as gender, technology, etc. Finally, the factors that can contribute to depression, such as physical, cultural factors, etc. In conclusion, depression is a disorder that both external and internal factors can contribute to.

\section{DEPRESSION INTRODUCTION: TEENAGER}

According to DSM 5, depression is a common and serious illness that negatively affects how you feel, think, and behave, such as eating, sleeping, and working [4]. According to the World Health Organization, depression is a common mental disorder that affects more than 264 million people worldwide [5].

The situation of people diagnosed with depression is getting worse each year. A study done by the China Youth and Children Research Center and the Institute of Psychology revealed that nearly $30 \%$ of young people between the ages of 14 to 35 have depression, while $8.1 \%$ are considered high risk [1]. The situation is getting worse compared to the study carried out in 2008. In 2008, $75.8 \%$ were free from depression. But in 2019 , only $70.5 \%$ show no depression tendencies, and $21.4 \%$ were in danger of becoming depressed. The experience divides people into three age groups, middle school students, university students, and office workers. The results show more middle school students are easily depressed and anxious compared to the other two groups. With a 5.5\% depress rate compare to $3.9 \%$ among university students and $5.2 \%$ among office workers [1].

As of the COVID 19 outbreak, the number of teenagers has risen. In a study, the Chinese researchers conducted an online survey of more than 8,000 middle school students aged 12 to 18 from more than 20 provinces in China in March 2020. These surveys include widely used questionnaires that are used worldwide to diagnose depression and anxiety disorders. Researchers found that $44 \%$ of Chinese students have mild to severe depression, and $37 \%$ have anxiety symptoms. More than $30 \%$ of students have symptoms of depression and anxiety at the same time. Previous research in China found that the average depression rate among children in the wider age group from kindergarten to grade 12 was $15 \%[6]$.

\subsection{Depression: Symptoms, diagnosis, and treatment}

When a person has depression, they will have these symptoms, which must happen for 2 weeks [4]: Such as depressed mood most of the day, almost every day.

Symptoms

- The interest or pleasure in all or almost all activities is significantly reduced for most of the day.

- Almost every day without diet or weight gain, or appetite decreased or increased, and the weight was significantly reduced

- Feeling worthless or excessive or inappropriate guilt almost every day.

- Repeated thoughts of death, repeated suicidal thoughts without a specific plan, or attempted suicide or had a specific suicide plan [4].

Diagnosis

- The doctor can diagnose depression based on:

- Physical exam: Take a physical exam and ask about your health. In some cases, depression may be related to some physical health problems.

- Lab tests: For example, a doctor may have a blood test called a complete blood count or test your thyroid to properly function.

- Psychological assessment: Mental health professionals will ask about your symptoms, thoughts, feelings, and behavior patterns. You may be asked to fill out a questionnaire to help answer these questions.

-DSM-5: The list of criteria in the DSM-5 book [4]

Treatment

- Medical treatment: Use medicine to reduce the symptoms of depression

- Psychotherapy: Psychotherapy is treating depression by discussing your condition and related problems with a mental health professional. Psychotherapy can help you:

- Adapt to crisis or other current difficulties

- Identify negative beliefs and behaviors and replace them with healthy, positive beliefs and behaviors

-Explore relationships and experiences, and build positive interactions with others

- Find better ways to deal with and solve problems

- Hospitalization: When the depression is very severe and requires hospitalization. This may be necessary if 
you cannot take care of yourself properly or in direct danger of harming yourself or others. Receiving psychiatric treatment in the hospital can help you stay calm and safe until your mood improves.

Other treatments such as electroconvulsive therapy (ECT). In ECT, the electric current passes through the brain to affect the function and effect of neurotransmitters in the brain, thereby alleviating depression. ECT is usually used for people who have poor drug treatment, cannot take antidepressants for health reasons, or are at high risk of suicide.

Transcranial magnetic stimulation (TMS). For those who do not respond to depression medicine, TMS may be an option. During TMS, a therapeutic coil placed on the scalp emits short magnetic pulses to stimulate nerve cells in the brain involved in mood regulation and depression [7].

\subsection{The impact of Depression on teenager}

Adolescence is an important developmental period, and they are building an understanding of relationships, explore their interests, and develop skills for their lives. The transition from education to the labor force [8]. Experiencing depression during this special period can disrupt these processes and ultimately affect one's longterm social and economic status, such as friendship and romantic relationships [9]. The early experienced depression also links to poor psychosocial outcomes, including unemployment, low education rate, and lower social support. All in all, understanding depression can impact a teenager can have long-term benefits for their psychology and society [10].

Adolescents who experience depression would have more frequent physical problems. And less attend school increases the risk of attempting suicide [11].

First, depression is often associated with functional impairment. Adolescents could experience negative effects on complete schoolwork and influence their school attendment, employment opportunities [12]. Second, depression can affect one's school attendance. This can affect educational attainment. Formation and maintaining relationships with people use less time with peers [12]. Third, depression is often marked as a social experiment, which is hard to develop stable relationships in the workplace and affects one's workability in the later future [13]. Fourth, depression could lead to various social issues, including a lower education rate, unemployment, and welfare [14]. And can add stress to the intimate relationship due to the financial restraint, lead to poor relationship function, and ultimate divorce [15]. So, studying how depression works is important because this can help us prevent the disorder rate that will happen in the future.

\section{DIFFERENCE OF PRESENCE OF DEPRESSION BETWEEN CHINA AND WESTERN COUNTRIES}

In the beginning, the present study would explore the status quo of Psychology applications in Chinese society and school and explain several factors related to the discrepancy. Next, the study would mainly introduce the identified special manifestation of depression in China. In addition, it would present the gender differences in depression that have been discovered by Chinese scientists and which have seldom been investigated by Western research. Finally, the study would provide unique treatments of depression that have been proven to be efficient in China.

\subsection{Cross-Cultural Perspectives on Depression: Concepts and Management}

Compared to Psychology applications in developed countries, one in China is restricted by limited professional and social support. Society faces a desperate shortage of psychiatrists, psychotherapy, psychological counseling, and social service. Firstly, China has an enormous total number of people who need psychotherapists. According to a journal from Shanghai China news in 2019, the psychotherapist gap reached up to 430,000 in China [16]. Although China has the largest population of 1.398 billion globally, approximately $16.6 \%$ of people in China have psychological disorders but seldom receive instant and appropriate treatments [17]. Secondly, professional psychological practitioners are few in number. For instance, Fang et al. (2020) state that compared to the U.S with general 200,000 psychologists, China only has 5000 psychologists [18].

Although the number of psychologists is increasing these years, only a fraction of them experience professional training like residency and obtain the qualification to prescribe drugs. In hospitals, nurses are shown to hold a high appreciation of the capability of adolescents' health and development (AHD). Still, they have limited knowledge and ability to help adolescents because of a lack of training [19]. The severity of the lack of a professional mental health workforce was highlighted during COVID-19 in China, especially for adolescents and people with lower socioeconomic status [18]. Despite the Chinese proclamation to strengthen the training of professional mental health workforce in 2015 , it is argued that the plan could not be done in a short period as there are still many obstacles existing in Chinese psychology as one of the most serious obstacles is that both psychiatrists and people with mental disorders are stigmatized in China [20]. The stigma may induce less help-seeking behaviors and a lower employment rate of psychiatrists. For instance, Liu et al. (2010) detect that families who have never sought help from psychotherapists believe that counseling is where 
psychiatrists treat mental illness. Only patients with serious mental illness would go [20]. The misconception of counseling hinders people with common mental disorders from accepting the help of psychotherapists.

Psychotherapy is also limited in school. It has been shown that school psychology is believed to be essential to children and family development. Still, there is no systematic program of school psychology initiated in Mainland China, which possesses the largest population of school-age children in 48 countries surveyed by the study [21]. Moreover, the expertise of existing school psychologists is questionable as Jimerson et al. find that despite the largest population of school-age children in 48 countries. Even though China only has 96 school psychologists, the official system gives no evidence of school psychologists in China after in-depth investigation. The crisis of professional school psychologists is much more serious than the lack of clinical psychiatrists. As Leung et al. (2008) estimate that the prevalence of mental disorders in Chinese adolescents is $16.4 \%$ which is mostly the same as $16.6 \%$ in Chinese people in 2019, the high rate of Chinese adolescents with mental disorders implies that adolescents also have heavy psychological burdens as adults do [22]. Among Chinese adolescents with mental disorders, the point prevalence of major depressive disorder (MDD) is $1.3 \%$ which is similar to the prevalence in other countries. Although China has the lowest suicidal rate (6.7) among Japan (12.2), South Korea (21.2), and the United States (14.5) in 2019 [23], the result interferes with adolescents' suicidal data from the survey that Chinese psychologists took from 2000 to 2013 aiming at suicidal-related behaviors among Chinese adolescents [24]. Dong et al. appeal for immediate attention to Chinese adolescents' mental health as they discover that the rate of suicide ideation is $17.7 \%$ among middle school adolescents, the rate of suicide plan is $7.3 \%$, and the suicide attempt is $2.7 \%$ [24]. Except for a general estimation of the number of adolescent suicidal events in each year, no official data has been provided. Few studies are aiming at whether adolescents who commit suicide have experienced or been diagnosed with depression, whether they have received psychotherapy, and whether depression significantly relates to Chinese adolescents' suicide rate, let alone whether present psychotherapy has high therapeutic efficacy to Chinese adolescents.

\subsection{Somatization of Depression}

Although in some previous studies, scientists find that the rate of adolescents with MDD is similar to the rates in other Eastern or Western countries, Parker et al. (2001) discover that Chinese people are prone to declare somatic symptoms (like insomnia and fatigue) rather than cognitive symptoms (like depressed mood and pessimism) after recalling their experience of seeking help [25]. However, Australian people are prone to cognitive symptoms. One explanation is that mental disorder conflicts with mental firmness, which Chinese culture prefers as having depressive moods represents emotionality and weak-will [25]. Throughout Chinese history, great fortitude and tenacious struggle have been promoted as the central spirit of the Communist Party of China (CPC). A Chinese informal expression of neurasthenia is also a swear word in daily life [26]. Therefore, stigma relates to mental illness itself more closely than counseling. Resistance to being viewed as weak or being associated with abusive words may lead to the formation of stigma in mental disorders in a vicious circle. The preference of the Chinese to mental firmness finally leads to the avoidance of acknowledging depression. As a result, the somatized expression of depression may be more culturally acceptable than the cognitive one in China [25]. The other explanation is the lack of psychological knowledge. Because of confining psychological development in China, many people have not fully understood what kind of feeling can be categorized as depressed mood, anxious mood, or low self-esteem. The misconception of cognitive feelings hampers their determination [25]. More research should be conducted to figure out whether the somatization of depression in China is intentional or not.

\subsection{Gender Difference and Correlative Factors}

\subsubsection{Gender Difference}

Gender difference which is formed by biological and cultural differences, leads to the disparate potentiality of having depression in teenage boys and teenage girls. Menarche and menstrual problems are risk factors of committing suicide in both Chinese and Western teenage girls. One disorder that only happens to females with comorbidity of depression is premenstrual dysphoric disorder (PMDD) (DSM-5). Although PMDD also happens to girls in the West, the rate of PMDD in East Asia is proven to be lower than the rate in the West [27].

Ussher's perspective (2003) can partially explain the difference as the psychologist believes that the creation of PMDD represents the subjectification of Women [28]. The natural part of women's physiological reaction is regarded as an illness that can be treated and eradicated. The popularization of PMDD in the West drives both men and women in Western society to discriminate against the female body and women's normal emotional fluctuation. The stereotype of women leads to an increasing rate of PMDD along with depression in western females.

\subsubsection{Correlative factors}

According to previous studies, cultural differences also enable academic pressure to affect teenage boys and teenage girls to varying degrees. Quach et al. (2013) indicate that even though authoritarian parenting style 
raises academic pressure in both boys and girls, the correlation between parental warmth and mental distress is relatively weaker for teenage girls than for teenage boys [29]. It can be interpreted that the traditional Chinese social norm of highlighting the male ability more than the female ability in the workplace may lead to higher sensitivity of teenage boys to their parents' emotional response.

However, gender discrimination engenders more teenage girls suffering from depression than teenage boys, while psychologists leave aside familial factors. Ample preceding studies show that eating disorders, body dissatisfaction, and fatphobia are more prevalent in both Chinese and western teenage girls than teenage boys [30]. It may be explained that ideal body image depending on male aesthetics is propagandized on social media because males dominate the most resources in Chinese society. Females are praised for being in a subordinate role. However, a prior study shows that boys and girls have disparate attitudes towards romantic involvement in China. Boys who have past experience of romantic relationships possess more confidence than presently dating or never-dated peers. However, girls' states are the opposite. Girls with past experience of romantic relationships possess less confidence than presently dating or never-dated peers [9]. It could be interpreted that boys tend to regard the number of romantic relationships as glory, but girls tend to regard it as shame. Nonetheless, little western research has probed the perception of the number of romantic relationships of teenage boys and girls.

Technology also influences girls and boys differently. For example, Liang et al. (2016) find that the causal relationship between internet addiction and depression has different directions in teenage boys and girls [31]. Teenagers are more prone to internet addiction because of depression, but teenage girls, are more prone to depression because of internet addiction. It is speculated that boys tend to access negative mood regulation through internet use, but girls are more sensitive to real social support. A similar conclusion is found in research in Taiwan, and female adolescents with depression have a higher potential of getting internet addiction in Korea [32]. Further research is needed to investigate the different impacts of internet use on boys and girls in the West.

\subsection{Treatment}

According to American Psychological Association (2019), medications like Serotonin-Selective Reuptake Inhibitors (SSRI), Serotonin/Norepinephrine-Reuptake inhibitors (SNRIs), and Bupropion are used to treat MDD. They usually combine with psychotherapy like Cognitive Behavioral Therapy (CBT) together to increase the efficiency of treatments [7]. Special treatments like herbal medicine, acupuncture, electro-acupuncture are proven to successfully treat Chinese MDD and pregnant women with MDD [33]. The mechanism of acupuncture on MDD should be investigated more, but the placebo effect may help patients with MDD feel better. However, many studies probing treatments of MDD suggest that the best way to decrease MDD is to prevent the development of MDD. Strengthening protective factors like familiar warmth, peer warmth, and social support has higher efficiency than avoiding risk factors, as protective factors foster a positive coping style and resilience. Several programs have been implemented in adolescent psychology in China. For example, Positive Youth Development (PYS) has led to greater life satisfaction in mainland Chinese adolescents [34]. The school-based program called "The Little Prince is Depressed" in Hong Kong has been tested with robust effectiveness and sustainability while it is led by school teachers [35]. Notably, searching for the meaning of life is an emerging protective factor to prevent MDD in China [36]. Therefore, social workers could design and organize group therapies, school activities, or company activities deriving from searching for the meaning of life to minimize the development of MDD in China. More studies should be aimed at the efficacy of special therapies of depression in the West.

\section{EXPLORE THE REASONS FOR THE DIFFERENCES}

Whether individual traits -- genes, personality -- play a role in developing depression has been debated in psychology for years. In fact, the cause of depression is very complex, there is no final conclusion on the cause of depression in medicine, but, certainly, genes, genetics, psychological and social environment, and many other factors lead to the occurrence of depression. Different diagnostic factors may also be present in different cultures.

In Chinese culture, people who show physical discomforts, such as dizziness or fatigue, may be diagnosed with depression because they think that psychological sadness and sadness are morally unacceptable [37]. In fact, in the DSM-5, depression is the mood of a long-term illness. Everyone experiences the emotion of depression, but different cultural environments present different symptoms and states of the disease [37]. Black women, for example, have lower rates of depression and suicide than white women, and immigrants have lower rates of depression than their offspring. These cultural influences may not be a dangerous cause of disease [37].

\subsection{Internal factors-- genes, heredity}

Genetic and environmental factors influence every disease. It's just a matter of weight. So does depression. There are over 100 million depression patients in the world. The severity of the disease is different. The 
performance is also different. In 2015, a team led by Professor Jonathan Flint of the University of Oxford identified two loci in the human genome that, for the first time, showed strong evidence of a link to depression, also known as major depressive disorder. The research team led by Professor Flint decided to collect samples in China because of the large number of depressed people there and many undiagnosed depression patients there. To reduce the study's inaccuracy, they limited patients to Han Chinese women with major depression. The results showed that the scientists found two depression-related mutations, both located on chromosome 10, one near SIRT1 and the other at the intron site of the LHPP gene [38]. This is the first time scientists have identified a gene strongly associated with depression. This proves that there is a genetic link. But that doesn't mean that just because one or both parents are depressed, the child will be depressed. In the research in 2010, Verhagen mentioned that in the subgroups stratified by ethnicity (Caucasian or Asian), neither the allele-based analysis nor the genotype-based analyses showed significant effects of the Val66Met polymorphism on Major Depressive Disorder(MDD) risk [39]. However, met allele frequency was higher in Asians in the presence of lower MDD prevalence rates than Caucasians [39]. Thus, there may be a genetic or DNA difference between Caucasians and Asians to affect depression factors.

\subsection{EXTERNAL FACTORS}

\subsubsection{Cultural Background: Individual vs. Collective}

Depression is a complex disease, and its etiology is not very clear. It is a psychological illness (seeking counseling) and a physical illness (taking medication as prescribed by a doctor). In addition, the social environment is also involved in the causes of depression. It may be that Asian cultural factors that discourage people from seeking medical help contribute to the low number of cases in China. Still, it also means that those who do seek medical help are suffering from more severe depression. It is possible that the majority of people diagnosed in China are severely depressed [38]. In Hwu's study in 1996, he shows that a low lifetime prevalence of MDD has been observed in the Chinese, Japanese, and Korean populations [40]. And while The lifetime prevalence of MDD is $1.5 \%$ in Taiwan and $2.9 \%$ in South Korea [41]. However, the data from Bijl mentioned the average prevalence in Western populations is $15-17 \%$ [42]. Therefore, Western populations have higher rates of diagnosed depression, but that doesn't mean that Western populations have more depression per capita than Eastern populations.

The prevalence of depression among adolescents varies by sex, age, race, and ethnicity. Teenagers living in different cultures have different levels of depression.
Filial piety, which is unique to China, may inhibit children's individual will to the extent that their selfesteem and ability to perceive society are reduced. When children feel positive signs from their parents, they will actively become filial, and this factor will influence their peers. But an authoritative form of filial piety -- one that inversely correlates children's self-esteem with their social abilities -- can lead children to feel guilty about their own incompetence [43].

\subsubsection{Family environment \& only child}

In the life of teenagers, they will experience a lot of pressure from school, family, and peers. These pressures can make them feel anxious and detached. Among them, as the leading person in the living environment of teenagers, family factors are crucial. When teenagers receive excessive family pressure, it will cause them to be unable to live a normal life, thus deepening their anxiety. These sources of stress are parents' quarrels and verbal and emotional abuse. Research shows that family conflict and emotional stability between parents are closely related to adolescent depression [44]. Among participants with a good relationship between parents, the prevalence of depression was only $13 \%$. In contrast, among participants with a bad relationship between parents, the prevalence of depression in their children was $29.2 \%$ [ 45$]$.

In China, due to the influence of the filial piety model, Chinese children are likely to have more conflicts and quarrels with their parents during adolescence [43]. Because children's subjective evaluation of their parents and understanding of filial piety will have important results on their own psychological regulation. If teenagers feel too much control from their parents, they will adjust their psychological state by having conflicts and quarrels with their parents [43].

Is there more pressure on children to live in families with only one child? Or will children suffer from depression because their parents give them all their attention? This hypothesis sounds reasonable because when adolescents are chronically stressed, the risk of depression is high. However, in Bin's research, he proposed that children who had no siblings did not show higher levels of fear, anxiety, or depression than children who had siblings. The negative effects of being an only child were no more pronounced for girls than for boys. Children of only children had lower levels of anxiety and depression than those with siblings, regardless of gender or age [46]. And this is not what we expected before.

\subsubsection{Reality factor (popularization of psychotherapy)}

Mental illness has been around for hundreds of years, but it is only in recent years that it has become increasingly well understood. Previously, people with 
mental illness were prone to negative reactions such as lower self-esteem and lower hopes due to the social stigma of mental illness [47]. They develop self-shame and self-contempt, which can lead to chronic stress [47]. Twenty years ago, people with mental illness were generally not accepted in society and remained excluded [47]. But now, the stereotypical effect on people with mental illness -- for example, the number of people who blame schizophrenia -- has dropped from 8 to 6 percent [48]. However, the social acceptance of mental patients is still not high, and the public is obviously hostile to mental patients [48].

Many people believe that people with mental illness are more dangerous. Although there is still no systematic and complete cure for mental illness, we can still start by removing social stigma and letting more people know and understand the world and suffering of people with mental illness. By reducing social stigma, people with mental illness will feel less burdened and will be able to freely admit their condition and receive treatment.

So far, however, the number of psychiatrists worldwide does not seem to be sufficient to cope with the needs of contemporary psychiatric patients. In Wilk's paper, he points out that at a time when patient demand is increasing, psychiatrists are becoming more stable, indicating that demand is outweighing supply [49]. Nevertheless, as society has become more aware of mental illness, the number of people working in the profession has increased. Despite efforts to expand the field in developing countries like China, psychology is becoming one of the most popular majors in American universities in developed countries like the United States. Perhaps one day, we can develop a cure for mental illness, making it less mysterious and less dangerous.

\section{CONCLUSION}

In conclusion, the purpose of this paper is to let people have a basic understanding of the depression and depression rate among Chinese teenagers. In addition, what factors have triggered teenagers to have depression and the impact it had on them. Throughout the review, we found out that multiple factors can contribute to teenagers having depression from a cultural perspective. The depression rate for teenagers increases throughout the year, and there are huge impacts for teenagers who have depression socially and economically. This review hopes that by comparing the differences of different characteristics, it can provide more complete data for the related depression researchers and the workers engaged in the related education industry to better understand and study the corresponding development. We hope that the information presented in this article will provide complete material for researchers to develop effective treatments for depression and to maintain a healthy life in the future.

\section{REFERENCES}

[1] Jun, liang. (2019). Depression threatens nearly 30 percent of China's youth. Depression threatens nearly 30 percent of China's youth - People's Daily Online. http://en.people.cn/n3/2019/0412/c900009566136.html.

[2] Cohen, A. B. (2009). Many forms of culture. American Psychologist, 64(3), 194-204. https://doi.org/10.1037/a0015308

[3] Gada, M. T. (1982). A Cross Cultural Study of Symptomatology of Depression - Eastern Versus Western Patients - International Journal of Social Psychiatry, 28(3), 195-202. https://doi.org/10.1177/002076408202800305

[4] Truschel, J. (2020, September 25). Depression Definition and DSM-5 Diagnostic Criteria. Psycom.net - Mental Health Treatment Resource Since 1996. https://www.psycom.net/depressiondefinition-dsm-5-diagnostic-criteria/.

[5] Health, W. (2020). Depression. World Health Organization. https://www.who.int/healthtopics/depression\#tab=tab_1.

[6] Barshay, J. (2020, October 26). Depression and anxiety rise among Chinese teens during coronavirus pandemic. The Hechinger Report. https://hechingerreport.org/proof-pointsdepression-and-anxiety-rise-among-chinese-teensduring-coronavirus-pandemic/.

[7] American Psychological Association (2019, February 16). Clinical Practice Guideline for the Treatment of Depression Across Three Age Cohorts. PsycEXTRA

Dataset. https://doi.org/10.1037/e505892019-001

[8] Arnett, J. J. (2001). Conceptions of the Transition to Adulthood: Perspectives From Adolescence Through Midlife. Journal of Adult Development. https://link.springer.com/article/10.1023/A:102645 0103225.

[9] Li, X., Huang, C.-Y. S., \& Shen, A. C.-T. (2019). Romantic involvement and adolescents' academic and psychosocial functioning in Chinese societies. Children and Youth Services Review, 96, 108-117. https://doi.org/10.1016/j.childyouth.2018.11.036

[10] K, N., \& Galambos, N. (2013). Social, demographic, and health outcomes in the 10 years following adolescent depression. The Journal of adolescent health : official publication of the Society for Adolescent Medicine. https://pubmed.ncbi.nlm.nih.gov/23499382/. 
[11] Wright, D. R., Katon, W. J., Ludman, E., McCauley, E., Oliver, M., Lindenbaum, J., \& Richardson, L. P. (2016). Association of Adolescent Depressive Symptoms With Health Care Utilization and PayerIncurred Expenditures. Academic pediatrics. https://www.ncbi.nlm.nih.gov/pmc/articles/PMC47 $15622 /$.

[12] Rohde \& Seeley \& Klein (2003). Psychosocial functioning of young adults who have experienced and recovered from major depressive disorder during adolescence. Journal of abnormal psychology.

https://pubmed.ncbi.nlm.nih.gov/12943014/.

[13] Sandberg-Thoma, S. E., \& Kamp Dush, C. M. (2014, February 1). Indicators of Adolescent Depression and Relationship Progression in Emerging Adulthood. Journal of marriage and the family. https://www.ncbi.nlm.nih.gov/pmc/articles/PMC38 98672/.

[14] Jonsson, U., Bohman, H., Hjern, A., Knorring, L. von, Olsson, G., \& Knorring, A.-L. von. (2020, April 16). Subsequent Higher Education After Adolescent Depression: A 15-Year Follow-Up Register Study: European Psychiatry. Cambridge Core.

https://www.cambridge.org/core/journals/europeanpsychiatry/article/abs/subsequent-higher-educat

[15] Jonsson \& Bohman \& Paaren (2021). Intimate relationships and childbearing after adolescent depression: a population-based 15 year follow-up study. Social psychiatry and psychiatric epidemiology.

https://pubmed.ncbi.nlm.nih.gov/20512560/.

[16] Chen, J. (2019, May 11). A huge shortage of psychological counselors in China. CNS. http://www.sh.chinanews.com.cn/yljk/2019-0511/56387.shtml

[17] Huang, Y., Wang, Y., Wang, H., Liu, Z., Yu, X., Yan, J., Yu, Y., Kou, C., Xu, X., Lu, J., Wang, Z., He, S., Xu, Y., He, Y., Li, T., Guo, W., Tian, H., Xu, G., Xu, X., ... Wu, Y. (2019). Prevalence of mental disorders in China: a cross-sectional epidemiological study. The Lancet Psychiatry, 6(3), 211-224. https://doi.org/10.1016/s22150366(18)30511-X

[18] Fang, M., Hu, S. X., \& Hall, B. J. (2020). A mental health workforce crisis in China: A pre-existing treatment gap coping with the COVID-19 pandemic challenges. Asian Journal of Psychiatry, 54, 102265. https://doi.org/10.1016/j.ajp.2020.102265

[19] Liu, T., Pan, Y., \& Zang, Y. (2011). Investigation on nurses' cognition of competency of adolescent health and development. Journal of Nursing Science(02), 51-54. https://doi.org/CNKI:SUN:HLXZ.0.2011-03-033

[20] Liu, Z., Fang, X., \& Lin, C. (2010). A Qualitative Research on Adolescents and Parents' Understanding of Counseling. Chinese Journal of Applied Psychology(02), 106-117. https://doi.org/10.3969/j.issn.10066020.2010 .02 .002

[21] D’Amato, R. C., van Schalkwyk, G. J., Zhao, B. Y., $\& \mathrm{Hu}$, J. (2013). Understanding the development of school psychology in Mainland China. School Psychology International, 34(2), 131-144. https://doi.org/10.1177/0143034312453392

[22] Leung, P. W., Hung, S.-fong, Ho, T.-pong, Lee, C.chiu, Liu, W.-sum, Tang, C.-pan, \& Kwong, S.leung. (2008). Prevalence of DSM-IV disorders in Chinese adolescents and the effects of an impairment criterion. European Child \& Adolescent Psychiatry, 17(7), 452-461. https://doi.org/10.1007/s00787-008-0687-7

[23] World Health Organization. (2021, June 16). Suicide worldwide in 2019. https://www.who.int/publications/i/item/97892400 26643

[24] Dong, Y.-ha, Liu, Y., Liu, L., He, W., Peng, G., Yin, Y., Chen, T., \& Mao, X. (2014). Reported Rate of Suicide-Related Behaviors among Chinese Adolescents: A Meta-Analysis. Chinese Journal of School Health, 35(4), 532-536. https://doi.org/CNKI:SUN:XIWS.0.2014-04-021

[25] Parker, G., Cheah, Y.-C., \& Roy, K. (2001). Do the Chinese somatize depression? A cross-cultural study. Social Psychiatry and Psychiatric Epidemiology, $36(6)$, 287-293. https://doi.org/10.1007/s001270170046

[26] Li, Y. (2021, July 1). Xi addresses ceremony marking CPC centenary. ECNS. http://www.ecns.cn/news/politics/2021-0701/detail-ihansikz8720597.shtml.

[27] Schatz, D. B., Hsiao, M.-C., \& Liu, C.-Y. (2012). Premenstrual Dysphoric Disorder in East Asia: A Review of the Literature. The International Journal of Psychiatry in Medicine, 43(4), 365-380. https://doi.org/10.2190/pm.43.4.f

[28] Ussher, J. M. (2003). The Role of Premenstrual Dysphoric Disorder in the Subjectification of Women. Journal of Medical Humanities, 24(1/2), 131-146. https://doi.org/10.1023/a:1021366001305

[29] Quach, A. S., Epstein, N. B., Riley, P. J., Falconier, M. K., \& Fang, X. (2013). Effects of Parental 
Warmth and Academic Pressure on Anxiety and Depression Symptoms in Chinese Adolescents. Journal of Child and Family Studies, 24(1), 106-116. https://doi.org/10.1007/s10826-013-9818-y

[30] Lai, C.-M., Mak, K.-K., Pang, J. S., Fong, S. S. M., Ho, R. C. M., \& Guldan, G. S. (2013). The associations of sociocultural attitudes towards appearance with body dissatisfaction and eating behaviors in Hong Kong adolescents. Eating Behaviors, 14(3), 320-324. https://doi.org/10.1016/j.eatbeh.2013.05.004

[31] Liang, L., Zhou, D., Yuan, C., Shao, A., \& Bian, Y. (2016). Gender differences in the relationship between internet addiction and depression: A crosslagged study in Chinese adolescents. Computers in Human Behavior, 63, 463-470. https://doi.org/10.1016/j.chb.2016.04.043

[32] Ha, Y.-M., \& Hwang, W. J. (2014). Gender Differences in Internet Addiction Associated with Psychological Health Indicators Among Adolescents Using a National Web-based Survey. International Journal of Mental Health and Addiction, 12(5), 660-669. https://doi.org/10.1007/s11469-014-9500-7

[33] Yang, Z., Zhao, L., Xie, X., Xu, T., Zhang, Y., Wang, X., Du, J., Wang, Z., Zhou, M., Li, Y., \& Zhou, S. (2017). The effectiveness of acupuncture for chronic pain with depression. Medicine, 96(47). https://doi.org/10.1097/md.0000000000008800

[34] Zhou, Z., Shek, D. T., \& Zhu, X. (2020). The Importance of Positive Youth Development Attributes to Life Satisfaction and Hopelessness in Mainland Chinese Adolescents. Frontiers in Psychology, 11. https://doi.org/10.3389/fpsyg.2020.553313

[35] Lai, E. S., Kwok, C.-L., Wong, P. W., Fu, K.-W., Law, Y.-W., \& Yip, P. S. (2016). The Effectiveness and Sustainability of a Universal School-Based Programme for Preventing Depression in Chinese Adolescents: A Follow-Up Study Using QuasiExperimental Design. PLOS ONE, 11(2). https://doi.org/10.1371/journal.pone.0149854

[36] Lew, B., Chistopolskaya, K., Osman, A., Huen, J. M., Abu Talib, M., \& Leung, A. N. (2020). Meaning in life as a protective factor against suicidal tendencies in Chinese University students. BMC Psychiatry, 20(1). https://doi.org/10.1186/s12888020-02485-4

[37] Kleinman, A. (2004). Culture and depression. New England Journal of Medicine, 351(10), 951-953.
[38] J. Flint,et al. Sparse whole-genome sequencing identifies two loci for major depressive disorder. Nature 2015, 523.doi:10.1038/nature14659.

[39] Verhagen, M., van der Meij, A., van Deurzen, P. et al. Meta-analysis of the BDNF Val66Met polymorphism in major depressive disorder: effects of gender and ethnicity. Mol Psychiatry 15, 260271 (2010). https://doi.org/10.1038/mp.2008.109

[40] Hwu, H. G., Chang, I. H., Yeh, E. K., Chang, C. J., \& Yeh, L. L. (1996). Major depressive disorder in Taiwan defined by the Chinese Diagnostic Interview Schedule. Journal of Nervous and Mental Disease.

[41] Weissman, M. M., Bland, R. C., Canino, G. J., Faravelli, C., Greenwald, S., Hwu, H. G., ... \& Yeh, E. K. (1996). Cross-national epidemiology of major depression and bipolar disorder. Jama, 276(4), 293 299.

[42] Bijl, R. V., Ravelli, A., \& Van Zessen, G. (1998). Prevalence of psychiatric disorder in the general population: results of The Netherlands Mental Health Survey and Incidence Study (NEMESIS). Social psychiatry and psychiatric epidemiology, 33(12), 587-595.

[43] Leung, A. N. M., Wong, S. S. F., Wong, I. W. Y., \& McBride-Chang, C. (2010). Filial piety and psychosocial adjustment in Hong Kong Chinese early adolescents. The Journal of Early Adolescence, 30(5), 651-667.

[44] Lewis A J, Kremer P, Douglas K (2015) Gender differences in adolescent depression: Differential female susceptibility to stressors affecting family functioning. Australian Journal of Psychology, 2015,67(3):131-138

[45] Ren Z, Zhou G, Wang Q (2019)Associations of family relationships and negative life events with depressive symptoms among Chinese adolescents: A cross-sectional study. Plos One, 2019, 14(7):e0219939

[46] Yang, B., Ollendick, T. H., Dong, Q., Xia, Y., \& Lin, L. (1995). Only children and children with siblings in the People's Republic of China: Levels of fear, anxiety, and depression. Child development, 66(5), 1301-1311.

[47] Rüsch, N., Müller, M., Lay, B. et al. Emotional reactions to involuntary psychiatric hospitalization and stigma-related stress among people with mental illness. Eur Arch Psychiatry Clin Neurosci 264, 3543 (2014). https://doiorg.ezproxy.uvm.edu/10.1007/s00406-013-0412-5

[48] Schomerus, G., Schwahn, C., Holzinger, A., Corrigan, P. W., Grabe, H. J., Carta, M. G., \& 
Angermeyer, M. C. (2012). Evolution of public attitudes about mental illness: A systematic review and meta-analysis. Acta Psychiatrica Scandinavica, 125(6), 440-452.

[49] Wilk, J. E., West, J. C., Narrow, W. E., Rae, D. S., \& Regier, D. A. (2005). Economic grand rounds: access to psychiatrists in the public sector and in managed health plans. Psychiatric Services, 56(4), 408-410. 